\title{
The effect of holmium laser resection versus standard transurethral resection on non-muscle- invasive bladder cancer: A systematic review and meta-analysis
}

\section{Changlong $\mathrm{Li}$}

Chongqing Medical University First Affiliated Hospital

\section{Liang Gao}

Chongqing Medical University First Affiliated Hospital

Jindong Zhang

Chongqing Medical University First Affiliated Hospital

Xiaokang Yang

Chongqing Medical University First Affiliated Hospital

Chuan Liu ( $\square$ liuchuan100@hospital.cqmu.edu.cn )

Chongqing Medical University First Affiliated Hospital https://orcid.org/0000-0002-6362-0261

\section{Research article}

Keywords: Non-muscle-invasive bladder cancer, Holmium laser, Transurethral resection, Meta-analysis

Posted Date: July 30th, 2019

DOI: https://doi.org/10.21203/rs.2.12172/v1

License: (c) (i) This work is licensed under a Creative Commons Attribution 4.0 International License.

Read Full License

Version of Record: A version of this preprint was published at Lasers in Medical Science on January 31st, 2020. See the published version at https://doi.org/10.1007/s10103-020-02972-w. 


\section{Abstract}

Purpose: To explore the advantages and limitations of holmium laser resection of the bladder tumor (HOLRBT) versus standard transurethral resection of the bladder tumor (TURBT) in the treatment of nonmuscle-invasive bladder cancer (NMIBC). Methods: The eligible studies were selected from the following databases: PubMed, Cochrane Library and Embase. Studies comparing HOLRBT and TURBT for patients with NMIBC were included. The outcomes of interest were time of operation, catheterization, and hospitalization, rates of recurrence and perioperative complications, including obturator nerve reflex, bladder perforation, bladder irritation and urethral stricture. Results of all data were compared and analyzed by Review Manager 5.3. Results: A total of 9 comparative studies were finally included for analysis. Pooled data demonstrated that HOLRBT could significantly reduce the time in catheterization and hospitalization, the rates of recurrence in 2 years of follow-up, obturator nerve reflex, bladder perforation, bladder irritation compared with those in TURBT, respectively. However, no significant difference could be found between HOLRBT and TURBT in the time of operation, rate of recurrence at 1year follow-up and urethral stricture. Conclusions: The results of our research showed that HOLRBT would be a better choice than TURBT for patients with NMIBC.

\section{Background}

Bladder cancer is the 10th most common cancer worldwide, with an estimated 549,000 new cases and 200,000 deaths in $2018^{[1]}$. According to the depth of invasion, bladder cancer can be divided into two types: non-muscle-invasive bladder cancer (NMIBC) and muscle-invasive disease, in which $75 \%$ of bladder cancer belongs to the former ${ }^{[2]}$. NMIBC is defined as the tumor is confined to the mucosa or submucosa of bladder.

Previously, the patients with NMIBC were usually treated by transurethral resection of the bladder tumor (TURBT) combined with intravesical chemotherapy or immunotherapy ${ }^{[3]}$, which was the "golden standard" according to the guideline of European Association of Urology ${ }^{[4]}$. However, the limitations of TURBT were gradually presented, such as the occurrence of obturator nerve reflex, bladder perforation, bladder irritation, and postoperative bleeding. To overcome these shortages, holmium laser resection of the bladder tumor (HOLRBT) was applied, which showed satisfactory outcomes, especially in tissue cutting, vaporization, and hemostasis ${ }^{[5]}$. The aim of our study was to compare the safety and effectiveness of HOLRBT and TURBT for patients with NMIBC.

\section{Methods}

\subsection{Search strategy}

This study was performed following the principle of preferred reporting items for systematic review and meta-analysis protocols (PRISMA) ${ }^{[6]}$. The databases, PubMed, Cochrane Library, EMBASE, were respectively searched using the term in title/abstract: "superficial bladder cancer", "non-muscle-invasive 
bladder cancer", "holmium laser", "transurethral resection" up to Mar. 2019. The reference lists from identified documents were also searched. To avoid data duplication, the latest report was used if multiple studies described the same population.

\subsection{Inclusion and exclusion criteria}

All comparative studies, including randomized controlled studies (RCT), cohort studies (CS), and casecontrol studies (CCS), focusing on HOLRBT and TURBT for patients with NMIBC were included. On the other hand, studies would be excluded if: 1) studies included patients with muscle-invasive-bladder cancer or distant metastasis. 2) no one of the following outcomes of interest were reported, including time of operation, catheterization, and hospitalization, rates of recurrence and complications, such as obturator nerve reflex, bladder perforation, bladder irritation and urethral stricture. 3) data of studies were not sufficient for a meta-analysis. 4) studies presented as conference abstracts.

\subsection{Data extraction:}

Data were extracted from these studies by 2 independent reviewers. If the two reviewers disagreed, a consensus was reached after a discussion between them. When studies divided the patients into $\geq 3$ groups, only comparison between HOLRBT and TURBT was extracted to keep the baselines as similar as possible. When articles were not written in English, they were translated for data extraction if possible.

\subsection{Outcomes of interest}

The outcomes of interest were time of operation, catheterization, and hospitalization, rates of recurrence and perioperative complications, including obturator nerve reflex, bladder perforation, bladder irritation and urethral stricture.

2.5 Quality assessment and statistical analysis:

The level of evidence of the included studies was graded according to the criteria by the Centre for Evidence-Based Medicine in Oxford, United Kingdom ${ }^{[7]}$. In addition, the quality of each included CS and CCS was evaluated by the Newcastle-Ottawa Scale (NOS). The NOS was a widely used qualityassessment method in meta-analysis ${ }^{[8]}$. In the modified Newcastle-Ottawa scale, a score of 1-9 stars was allocated, and more stars stood for higher quality.

The meta-analysis was performed using Review Manager 5.3. The odd ratio (OR) and mean difference (MD) were used to describe results of dichotomous and continuous variables, respectively. All results were reported with $95 \%$ confidence intervals $(\mathrm{Cl})$. The $\mathrm{P}<0.05$ was regarded to be statistically significant.

In addition, statistical heterogeneities among trials were evaluated by $\mathrm{I}^{2}$ and $\chi^{2}$ tests. $I^{2}$ value of $25 \%$, $50 \%$ and $75 \%$ corresponded to low, medium and high levels of heterogeneity, respectively. When $\mathrm{I}^{2}<50 \%$ and $P>0.10$, a fixed-effect model was used. Otherwise, the random-effect model was applied. 


\section{Results}

\subsection{Study selection}

Through the search strategy, a total of 317 articles were initially found. After software and manual checking, 85 papers were excluded because of duplications. Then, 203 documents were excluded after screening of the title and abstract. Of the 29 remaining records, 20 articles were excluded after evaluation of the full-text. Finally, 9 studies ${ }^{[9-17]}$ with a total of 1,166 patients were included in the meta-analysis, in which 473 patients were treated by HOLRBT and 693 patients were treated by TURBT (Fig. 1). The characteristics of all available studies were shown in Table 1.

Totally, 1 RCT, 3 prospective CS and 5 retrospective CCS were included. For the postoperative intravesical instilled chemotherapy, 4 studies used mitomycin C, 2 used epirubicin, 2 used pirarubicin and 1 study did not describe the chemotherapy scheme. Five studies ${ }^{[9-11,15,17]}$ described a comparison of $\geq 3$ groups, from which only data describing HOLRBT and TURBT for NMIBC were extracted. One study was published in Chinese, but had an English abstract ${ }^{[12]}$.

\subsection{Outcomes of interest:}

\subsubsection{Operation time}

Eight studies including a total of 1,142 patients ${ }^{[9-12,14-17]}$ had evaluated data of operation time, which revealed that there was no significant difference in operation time between two groups (MD $=-0.76,95 \%$ Cl [-3.10, 1.58], $\mathrm{P}=0.53)$. (Fig. 2).

\subsubsection{Catheterization time}

When catheterization time was compared, 8 studies including 1,142 patients ${ }^{[9-12,14-17]}$ were analyzed. Pooled data demonstrated a significantly less time in catheterization time in the HOLRBT group compared to TURBT group (MD = -1.02, 95\% Cl [-1.35, -0.68], $\mathrm{P}<0.00001)$. (Fig. 3)

\subsubsection{Hospitalization time}

Seven studies including 1,002 patients ${ }^{[9-11,14-17]}$ are pooled, which indicated that hospitalization time was significantly less in HOLRBT group compared to TURBT group (MD $=-1.11,95 \% \mathrm{Cl}[-1.65,-0.58], \mathrm{P}$ $<0.0001$ ). (Fig. 4)

\subsubsection{1-year recurrence}

Data describing the rate of 1 -year recurrence were pooled from 5 studies ${ }^{[12-14,16,17]}$ of 493 patients. Result of meta-analysis showed that there was an insignificant difference between two groups ( $O R=0.72 ; 95 \%$ $\mathrm{Cl}[0.45,1.16], \mathrm{P}=0.18)$. Considering the large impact of different chemotherapy schemes to recurrence, 
we carried out a subgroup analysis, which showed that there was an insignificance in mitomycin C, epirubicin, pirarubicin and subgroups not mentioned chemotherapy scheme. (Fig. 5)

\subsubsection{2-year recurrence}

When the rate of 2-year recurrence were compared between two groups, pooled data from 8 studies including a total of 939 patients ${ }^{[9-10,12-17]}$ revealed that the recurrence was significantly higher in XX group $(\mathrm{OR}=0.69 ; 95 \% \mathrm{Cl}[0.51,0.94], \mathrm{P}=0.02)$. However, in the subgroup analysis, all results became insignificant in mitomycin $\mathrm{C}$, epirubicin and pirarubicin subgroups and in the subgroup did not describe chemotherapy scheme. (Fig. 6).

\subsubsection{Complications}

Four studies ${ }^{[10,12,15,17]}$ evaluated the incidence of obturator nerve reflex, which revealed significantly lower occurrence in the HOLRBT group (OR $=0.06 ; 95 \% \mathrm{Cl}[0.01,0.23], \mathrm{P}<0.0001)$. (Fig. 7)

Additionally, seven studies including 860 patients ${ }^{[9-10,12,14-17]}$ were pooled, which presented that the incidence of bladder perforation was significant lower in the HOLRBT group (OR $=0.15 ; 95 \% \mathrm{Cl}[0.05$, 0.45], $P=0.0008$ ). (Fig. 8)

What's more, six studies ${ }^{[9-10,12,14-16]}$ including evaluated data of bladder irritation, and a significance between two groups were observed ( $\mathrm{OR}=0.45 ; 95 \% \mathrm{Cl}[0.31,0.66], \mathrm{P}<0.0001)$. (Fig. 9)

When urethral stricture was compared between HOLRBT and TURBT groups, 4 studies ${ }^{[10,12,15-16]}$ including 444 patients were pooled, the result of meta-analysis showed there was an insignificance in urethral stricture between two groups $(\mathrm{OR}=0.86,95 \% \mathrm{Cl}[0.37,1.98], \mathrm{P}=0.73)$. (Fig. 10)

\subsection{Sensitivity analysis and publication bias}

To analyze the potential publication bias, a funnel plot of operation time was performed, which revealed that 2 studies had potential publication bias. After the studies were excluded, similar results in all outcomes of interest were showed. (Fig. 11)

\section{Discussion}

Because of the heavy burden of NMIBC, it became a key issue that how to improve the survival and quality of patients' life. At present, TURBT still played an important role in the diagnosis and treatment of NMIBC, because it could provide adequate tissue for pathological examination followed with all visible tumors being effectively removed ${ }^{[18,19]}$. However, the limitations of TURBT should not be ignored as well. Firstly, the occurrence of obturator nerve reflex was frequent during TURBT, especially for tumors at the lateral wall of bladder, possibly leading to bladder perforation ${ }^{[20]}$. In order to avoid this risk, inadequate resection depth of the tumors might be occurred ${ }^{[21]}$. Secondly, as probably larger thermal injury by TURBT, the time of catheterization, and hospitalization after operation might be longer. 
Fortunately, after laser was firstly applied in urology in $1978{ }^{[22]}$, the holmium laser was gradually used in the resection of bladder tumors since $2001^{[23]}$, which presented satisfactory outcomes. It made a necessary to compare these two technologies.

Our results showed that the time of catheterization and hospitalization was significantly shorter in the HOLRBT group than that in the TURBT group, but there was no significant difference in operation time.

The same conclusions were reported by Teng et al ${ }^{[24]}$. For the time of catheterization and hospitalization, because of thermal damage caused by TURBT, rapidly postoperative recovery could be showed after HOLRBT. However, significant heterogeneities among studies could be found, thus the accuracy of results would be largely affected.

Our study revealed that HOLRT had a significant benefit in 2-year recurrence compare with TUBRT, which could be calculated to be $26.1 \%$ in HOLRBT group and 34.3\%in TURBT group, respectively. In addition, rate of 1-year recurrence was insignificant. Considering the difference of postoperative intravesical instilled chemotherapy, subgroup analyses were carried out. Though postoperative adjuvant intravesical chemotherapy was an important factor affecting the recurrence of cancer ${ }^{[25]}$, no significant difference was found in our study. However, more evidence should be discovered to verify this conclusion.

Our study further confirmed the incidences of several perioperative complications, including obturator nerve reflex, bladder perforation, bladder irritation, which presented significant advantages in HOLRBT group. The rate of obturator nerve reflex, bladder perforation, bladder irritation could be calculated to be $0 \%, 0.2 \%, 22.0 \%$ in HOLRBT group and $11.2 \%, 5.3 \%, 39.1 \%$ in TURBT group, respectively. And, our study showed that there was an insignificance in the rate of urethral stricture between two groups, which could be calculated to be $4.8 \%$ in HOLRBT group and $5.8 \%$ in TURBT group, respectively. Based on these results, it seemed that HOLRBT would be safer than TURBT for patients with NMIBC.

Above all, there were still some limitations should be taken into consideration in this study. Firstly, only one RCT were included for analysis, which would lower the quality of pooled results. Secondly, some important parameters were not analyzed because the description of them could not be uniformed, such as pre- and postoperative bleeding. Thirdly, high heterogeneity could be observed in outcomes of operation time, catheterization time, and hospitalization time. These might be due to the differences of patient's condition, surgeons' technique and custom, etc.

\section{Conclusions}

Based on the results of our meta-analysis, HOLRBT would be superior to TURBT in safety and be similar in effectiveness for patients with NMIBC though sporadical insignificance between two groups were found. However, more high-quality clinical trials were needed to further confirm our conclusions. NOS:Newcastle-Ottawa Scale,

\section{Abbreviations}


HOLRBT: holmium laser resection of the bladder tumor, TURBT: transurethral resection of the bladder tumor, NMIBC: non-muscle-invasive bladder cancer, PRISMA: preferred reporting items for systematic review and meta-analysis protocols, RCT: randomized controlled studies, CS: cohort studies, CCS: casecontrol studies, OR: odd ratioand, MD: mean difference, Cl: confidence intervals. LOE: level of evidence, NA= not available.CL1: Chuan Liu, CL2: Changlong Li, GL: Gao Liang, JZ: Jindong Zhang, XY: Xiaokang Yang.

\section{Declarations}

\section{Ethics approval and consent to participate}

Ethical approval: All procedures performed in studies involving human participants were in accordance with the ethical standards of the institutional and/or national research committee and with the 1964 Helsinki declaration and its later amendments or comparable ethical standards.

Informed consent: Informed consent was obtained from all individual participants included in the study.

\section{Consent for publication}

Not applicable

\section{Availability of data and material}

The datasets used and/or analysed during the current study are available from the corresponding author on reasonable request.

\section{Competing interests}

Conflict of Interest: $\mathrm{CL}^{1}$ declares that he has no conflict of interest. $\mathrm{CL}^{2}$ declares that he has no conflict of interest. LG declares that he has no conflict of interest. XY declares that she has no conflict of interest. JZ declares that she has no conflict of interest

\section{Funding}

Not applicable

\section{Author contribution}

$\mathrm{CL}^{1}$ : Methodology

$\mathrm{CL}^{2} \llbracket$ Data Collection, Manuscript writing

LG $₫$ Data Collection, Manuscript writing

JZ: Writing - review \& editing 
$X Y$ : Writing - review \& editing

All authors read and approved the manuscript.

\section{Acknowledgements}

Not applicable

\section{References}

1. Bray F, Ferlay J, Soerjomataram I, Siegel RL, Torre LA, Jemal A (2018) Global cancer statistics 2018: GLOBOCAN estimates of incidence and mortality worldwide for 36 cancers in 185 countries. CA: a cancer journal for clinicians 68 (6):394-424. doi:10.3322/caac.21492.

2. Burger M, Catto JW, Dalbagni G, et al (2013) Epidemiology and risk factors of urothelial bladder cancer. Eur Urol 63 (2):234-241. doi:10.1016/j.eururo.2012.07.033.

3. Babjuk M, Bohle A, Burger M, et al (2017) EAU Guidelines on Non-Muscle-invasive Urothelial Carcinoma of the Bladder: Update 2016. Eur Urol 71 (3):447-461.

4. Kaufman DS, Shipley WU, Feldman AS (2009) Bladder cancer. Lancet (London, England) 374 (9685):239-249. doi:10.1016/s0140-6736(09)60491-8.

5. Zarrabi A, Gross AJ (2011) The evolution of lasers in urology. Ther Adv Urol 3 (2):81-89. doi:10.1177/1756287211400494.

6. Moher D, Liberati A, Tetzlaff J, Altman DG (2010) Preferred reporting items for systematic reviews and meta-analyses: the PRISMA statement. Int J Surg (London, England) 8 (5):336-341. doi:10.1016/j.ijsu.2010.02.007.

7. Phillips B (2004) GRADE: levels of evidence and grades of recommendation. Arch Dis Child 89 (5):489

8. Stang A (2010) Critical evaluation of the Newcastle-Ottawa scale for the assessment of the quality of nonrandomized studies in meta-analyses. Eur J Epidemiol 25 (9):603-605. doi:10.1007/s10654010-9491-z.

9. Chen GF, Shi TP, Wang BJ, Wang XY, Zang Q (2015) Efficacy of different resections on non-musleinvasive bladder cancer and analysis of the optimal surgical method. J Biol Regul Homeost Agents 29 (2):465-470.

10. Huang JH, Hu YY, Liu M, Wang GC, Peng B, Yao XD (2016) Comparative study of 2 um laser versus holmium laser for the resection of non-muscle invasive bladder cancer. Int $\mathrm{J}$ Clin Exp Med 9 (12):23618-23623

11. Kramer MW, Rassweiler JJ, Klein J, et al (2015) En bloc resection of urothelium carcinoma of the bladder (EBRUC): a European multicenter study to compare safety, efficacy, and outcome of laser and electrical en bloc transurethral resection of bladder tumor. WORLD J UROL 33 (12):1937-1943. doi:10.1007/s00345-015-1568-6 
12. Luo Y, Cao ZG, Liu J, Su JH, Zhu JG, Xu Z (2008) A comparison of clinical outcome between holmium laser resection and transurethral resection for superficial bladder carcinoma. Tumor 28 (11):1001-1003. doi:10.3781/j.issn.1000-7431.2008.11.022.

13. Muraro GB, Grifoni R, Spazzafumo L (2005) Endoscopic therapy of superficial bladder cancer in highrisk patients: Holmium laser versus transurethral resection. Surg Technol Int 14:222-226.

14. Verma A, D'Souza N (2016) Holmium laser transuretheral reserction of bladder tumor: Our experience. Indian J Urol 32.

15. Xishuang S, Deyong Y, Xiangyu C, et al (2010) Comparing the safety and efficiency of conventional monopolar, plasmakinetic, and holmium laser transurethral resection of primary non-muscle invasive bladder cancer. J ENDOUROL 24 (1):69-73. doi:10.1089/end.2009.0171.

16. Zhong C, Guo S, Tang Y, Xia S (2010) Clinical observation on 2 micron laser for non-muscle-invasive bladder tumor treatment: Single-center experience. WORLD J UROL 28 (2):157-161. doi:10.1007/s00345-010-0532-8.

17. Zhu Y, Jiang X, Zhang J, Chen W, Shi B, Xu Z (2008) Safety and Efficacy of Holmium Laser Resection for Primary Nonmuscle-Invasive Bladder Cancer Versus Transurethral Electroresection: Single-Center Experience. Urology 72 (3):608-612. doi:10.1016/j.urology.2008.05.028.

18. Babjuk M, Bohle A, Burger M, et al (2017) EAU Guidelines on Non-Muscle-invasive Urothelial Carcinoma of the Bladder: Update 2016. Eur Urol 71 (3):447-461. doi:10.1016/j.eururo.2016.05.041.

19. Herrmann TR, Liatsikos EN, Nagele U, Traxer O, Merseburger AS (2012) EAU guidelines on laser technologies. Eur Urol 61 (4):783-795. doi:10.1016/j.eururo.2012.01.010.

20. Khorrami MH, Javid A, Saryazdi H, Javid M (2010) Transvesical blockade of the obturator nerve to prevent adductor contraction in transurethral bladder surgery. J Endourol 24 (10):1651-1654. doi:10.1089/end.2009.0659

21. Mariappan P, Zachou A, Grigor KM (2010) Detrusor muscle in the first, apparently complete transurethral resection of bladder tumour specimen is a surrogate marker of resection quality, predicts risk of early recurrence, and is dependent on operator experience. Eur Urol 57 (5):843-849. doi:10.1016/j.eururo.2009.05.047.

22. Staehler G, Schmiedt E, Hofstetter A (1978) [Destruction of bladder neoplasms by means of transurethral neodym-YAG-laser coagulation]. Helv Chir Acta 45 (3):307-311.

23. Saito S (2001) Transurethral en bloc resection of bladder tumors. J Urol 166 (6):2148-2150.

24. Teng JF, Wang K, Yin L, Qu FJ, Zhang DX, Cui XG, Xu DF (2013) Holmium laser versus conventional transurethral resection of the bladder tumor. Chin Med J (Engl) 126 (9):1761-1765.

25. Oosterlinck W, Decaestecker K (2018) Update on early instillation of chemotherapy after transurethral resection of non-muscle-invasive bladder cancer. Expert Rev Anticancer Ther 18 (5):437-443. doi:10.1080/14737140.2018.1451748.

\section{Figures}




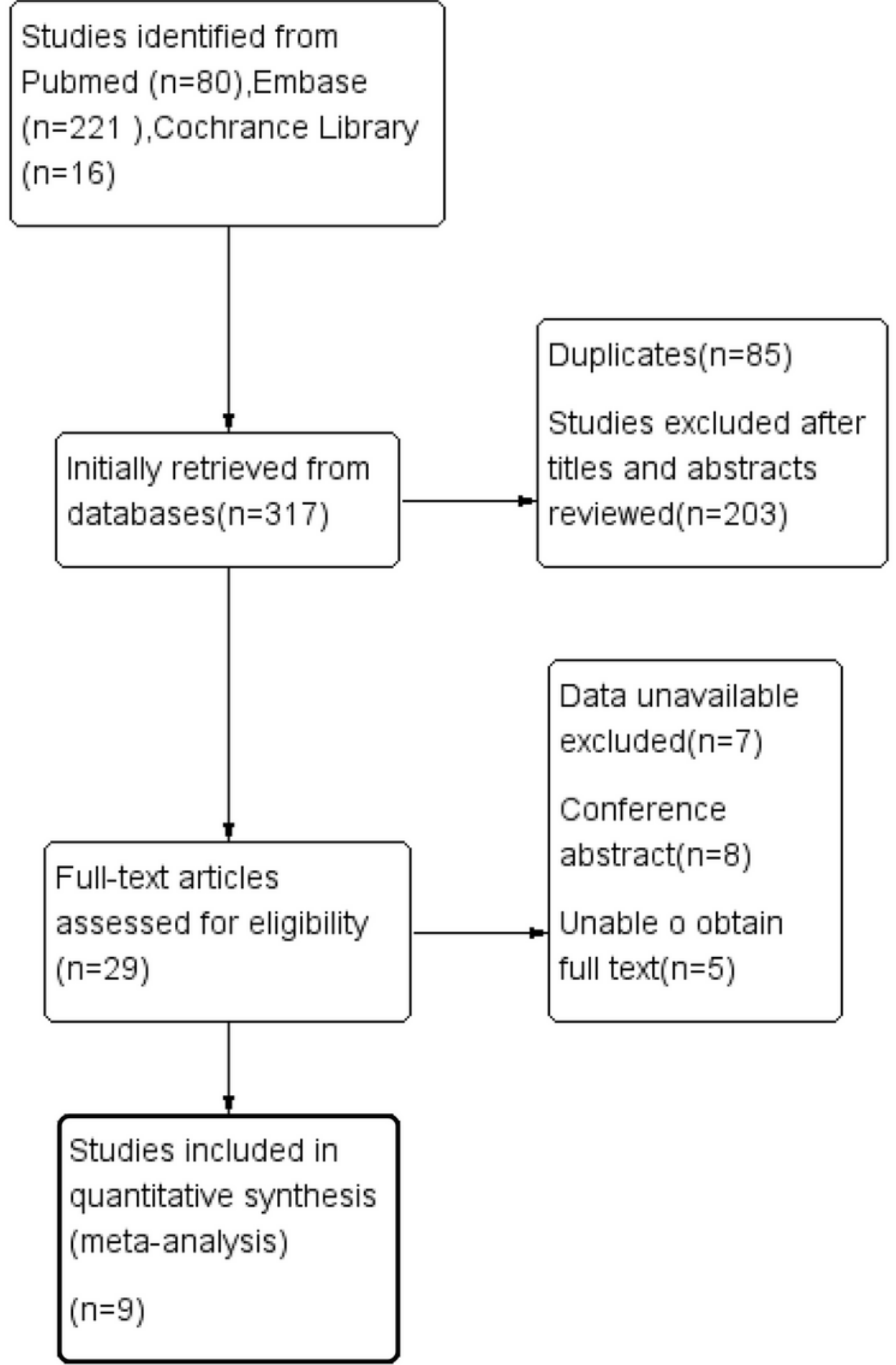

Figure 1

Flow diagram of study 


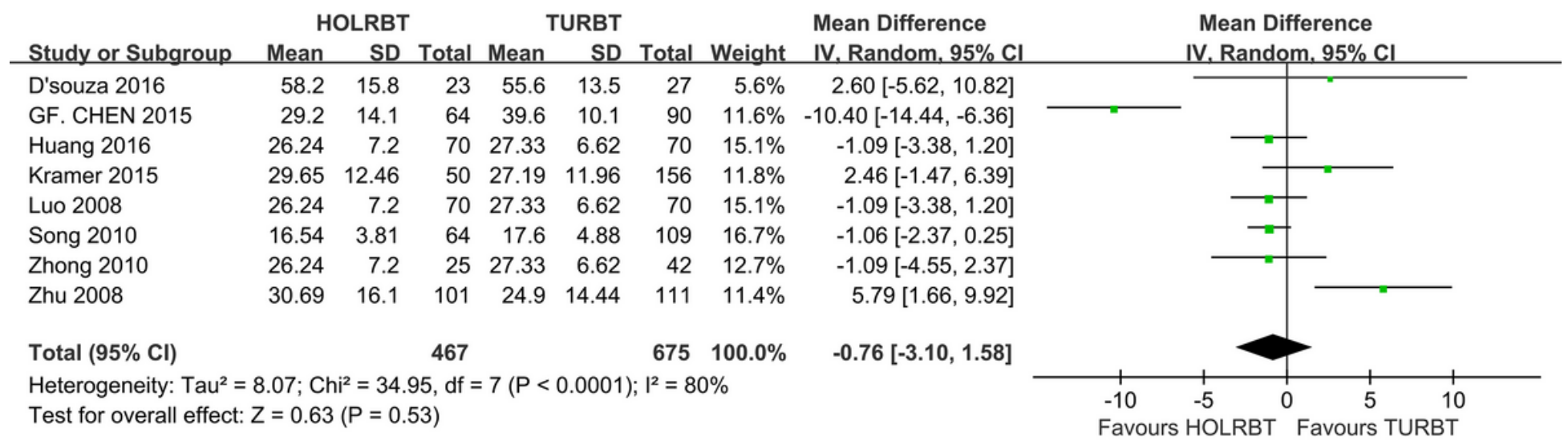

Figure 2

The results of operation time in Meta-analysis

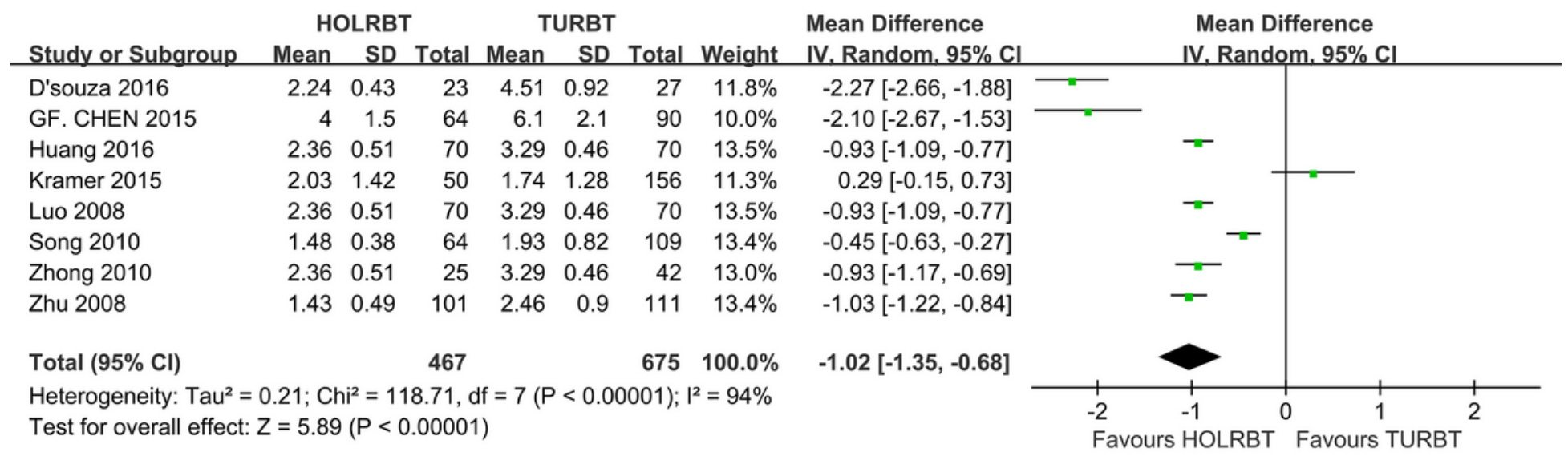

\section{Figure 3}

The results of catheterization time in Meta-analysis

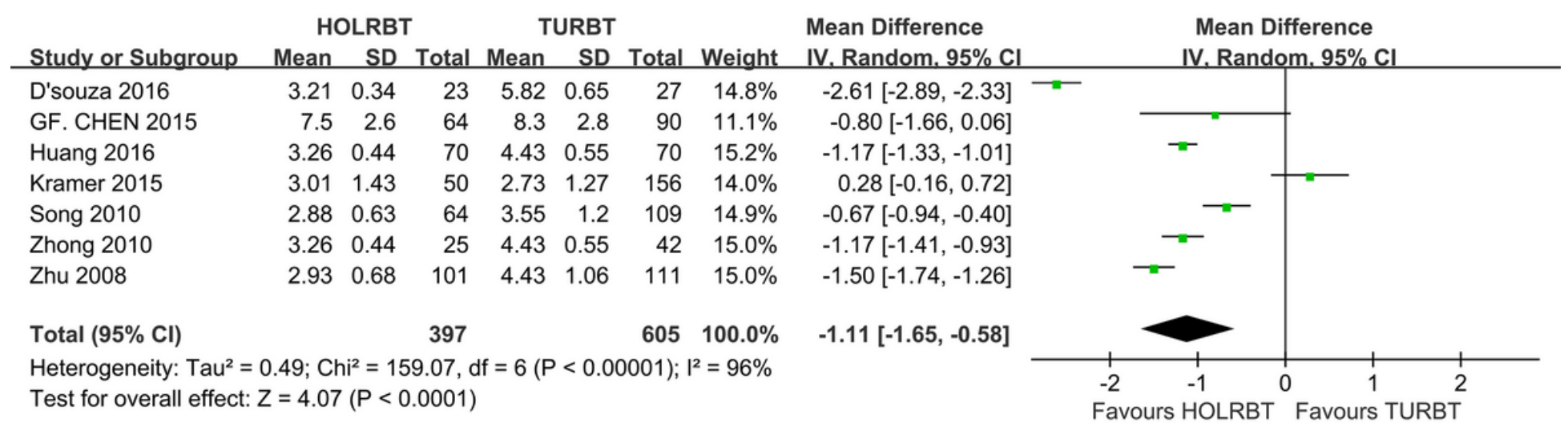

\section{Figure 4}

The results of hospitalization time in Meta-analysis 
HOLRBT

TURBT

Odds Ratio

Odds Ratio

Study or Subgroup

Events Total Events Total Weight M-H. Fixed, $95 \% \mathrm{Cl}$

1.8.1 mitomycin C

D'souza 2016

Zhu 2008

223

$19 \quad 101$

$3 \quad 27 \quad 6.1 \%$

$0.76[0.12,5.01]$

Subtotal $(95 \% \mathrm{CI})$

Total events

2130

Heterogeneity: Chi $^{2}=0.00, d f=1(P=0.96) ;\left.\right|^{2}=0 \%$

Test for overall effect: $Z=1.01(P=0.31)$

\subsection{2 epirubicin}

Zhong 2010

Subtotal $(95 \% \mathrm{Cl})$

25

$7 \quad 42 \quad 11.1 \%$

$0.68[0.16,2.92]$

Total events

3

7

Heterogeneity: Not applicable

Test for overall effect: $Z=0.52(P=0.61)$

1.8.3 pirarubicin

Luo 2008

Subtotal $(95 \% \mathrm{Cl})$

$2 \quad 26$

6

$38 \quad 10.9 \%$

$0.44[0.08,2.40]$

Total events

2

6

Heterogeneity: Not applicable

Test for overall effect: $Z=0.94(P=0.35)$

\subsection{4 not mentioned}

Muraro 2005

Subtotal $(95 \% \mathrm{Cl})$

$10 \quad 50$
$\quad 50$

11

$50 \quad 21.3 \%$

$0.89[0.34,2.32]$

$0.72[0.37,1.40]$

$0.73[0.39,1.35]$

Total events

10

11

Heterogeneity: Not applicable

Test for overall effect: $Z=0.25(P=0.81)$

Total $(95 \% \mathrm{Cl})$

Total events

Heterogeneity: $\mathrm{Chi}^{2}=0.50, \mathrm{df}=4(\mathrm{P}=0.97) ; \mathrm{I}^{2}=0 \%$

Test for overall effect: $Z=1.34(P=0.18)$

Test for subgroup differences: $\mathrm{Chi}^{2}=0.50, \mathrm{df}=3(\mathrm{P}=0.92), \mathrm{I}^{2}=0 \%$
$0.89[0.34,2.32]$

\section{Figure 5}

The results of 1-year recurrence in Meta-analysis 
Study or Subgroup Events Total Events Total Weight M-H. Fixed, 95\% C

1.6.1 mitomycin C

D'souza 2016

Song 2010

Zhu 2008

Subtotal $(95 \% \mathrm{Cl})$

Total events

$4 \quad 23$

$20 \quad 63$

33101

187

57

Heterogeneity: $\mathrm{Chi}^{2}=0.10, \mathrm{df}=2(\mathrm{P}=0.95) ; \mathrm{I}^{2}=0 \%$

Test for overall effect: $Z=1.74(P=0.08)$

\subsection{2 epirubicin}

Huang 2016

Zhong 2010

Subtotal $(95 \% \mathrm{Cl})$

862

$6 \quad 25$

87

$\begin{array}{rrr}7 & 27 & 4.8 \% \\ 44 & 107 & 20.0 \% \\ 44 & 111 & 25.4 \% \\ & 245 & 50.2 \%\end{array}$
95

Total events 14

62
25

$\begin{array}{rrr}9 & 60 & 7.2 \% \\ 13 & 42 & 6.6 \% \\ & 102 & 13.8 \%\end{array}$

$0.84[0.30,2.34]$

$0.70[0.23,2.17]$

$0.77[0.36,1.65]$
$0.60[0.15,2.39]$

$0.74[0.42,1.30]$

$0.70[0.46,1.05]$

Heterogeneity: $\mathrm{Chi}^{2}=0.05, \mathrm{df}=1(\mathrm{P}=0.82) ; \mathrm{I}^{2}=0 \%$

Test for overall effect: $Z=0.66(P=0.51)$

\subsection{3 theprubicine}

$\begin{array}{lrrrrrr}\text { GF. CHEN 2015 } & 21 & 64 & 37 & 90 & 18.6 \% & 0.70[0.36,1.37] \\ \text { Luo 2008 } & 3 & 26 & 9 & 38 & 5.8 \% & 0.42[0.10,1.73] \\ \text { Subtotal (95\% CI) } & & 90 & & \mathbf{1 2 8} & \mathbf{2 4 . 4 \%} & \mathbf{0 . 6 3}[\mathbf{0 . 3 5}, \mathbf{1 . 1 6}] \\ \text { Total events } & 24 & & 46 & & & \end{array}$

Heterogeneity: Chi $^{2}=0.41, \mathrm{df}=1(\mathrm{P}=0.52) ; \mathrm{I}^{2}=0 \%$

Test for overall effect: $Z=1.49(P=0.14)$

\subsection{4 not mentioned}

$\begin{array}{lcccccc}\text { Muraro } 2005 & 14 & 50 & 18 & 50 & 11.7 \% & 0.69[0.30,1.61] \\ \text { Subtotal }(\mathbf{9 5 \%} \mathrm{Cl}) & & \mathbf{5 0} & & \mathbf{5 0} & \mathbf{1 1 . 7 \%} & \mathbf{0 . 6 9}[\mathbf{0 . 3 0}, \mathbf{1 . 6 1}] \\ \text { Total events } & 14 & & 18 & & & \end{array}$

Heterogeneity: Not applicable

Test for overall effect: $Z=0.86(P=0.39)$

$\begin{array}{llcrrr}\text { Total }(95 \% \mathrm{Cl}) & 414 & & 525 & 100.0 \% & 0.69[0.52,0.92] \\ \text { Total events } & 109 & & 181 & & \end{array}$

Heterogeneity: $\mathrm{Chi}^{2}=0.72, \mathrm{df}=7(\mathrm{P}=1.00) ; \mathrm{I}^{2}=0 \%$

Test for overall effect: $Z=2.50(P=0.01)$

Test for subgroup differences: $\mathrm{Chi}^{2}=0.17, \mathrm{df}=3(\mathrm{P}=0.98), \mathrm{I}^{2}=0 \%$

\section{Figure 6}

The results of 2-year recurrence in Meta-analysis

HOLRBT TURBT

Study or Subgroup

Huang 2016

Luo 2008

Song 2010

Zhu 2008

Total $(95 \% \mathrm{CI})$

Total events

Heterogeneity: $\mathrm{Chi}^{2}=0.24, \mathrm{df}=3(\mathrm{P}=0.97) ; \mathrm{I}^{2}=0 \%$

Test for overall effect: $Z=3.95(P<0.0001)$

37

Odds Ratio Odds Ratio

M-H, Fixed, $95 \% \mathrm{Cl}$ M-H, Fixed, $95 \% \mathrm{Cl}$

$0.04[0.00,0.64]$

$0.05[0.00,0.80]$

0.09 [0.01, 1.63]

$0.07[0.00,1.22]$

$0.06[0.01,0.23]$

$328 \quad 100.0 \%$

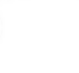

\section{Figure 7}




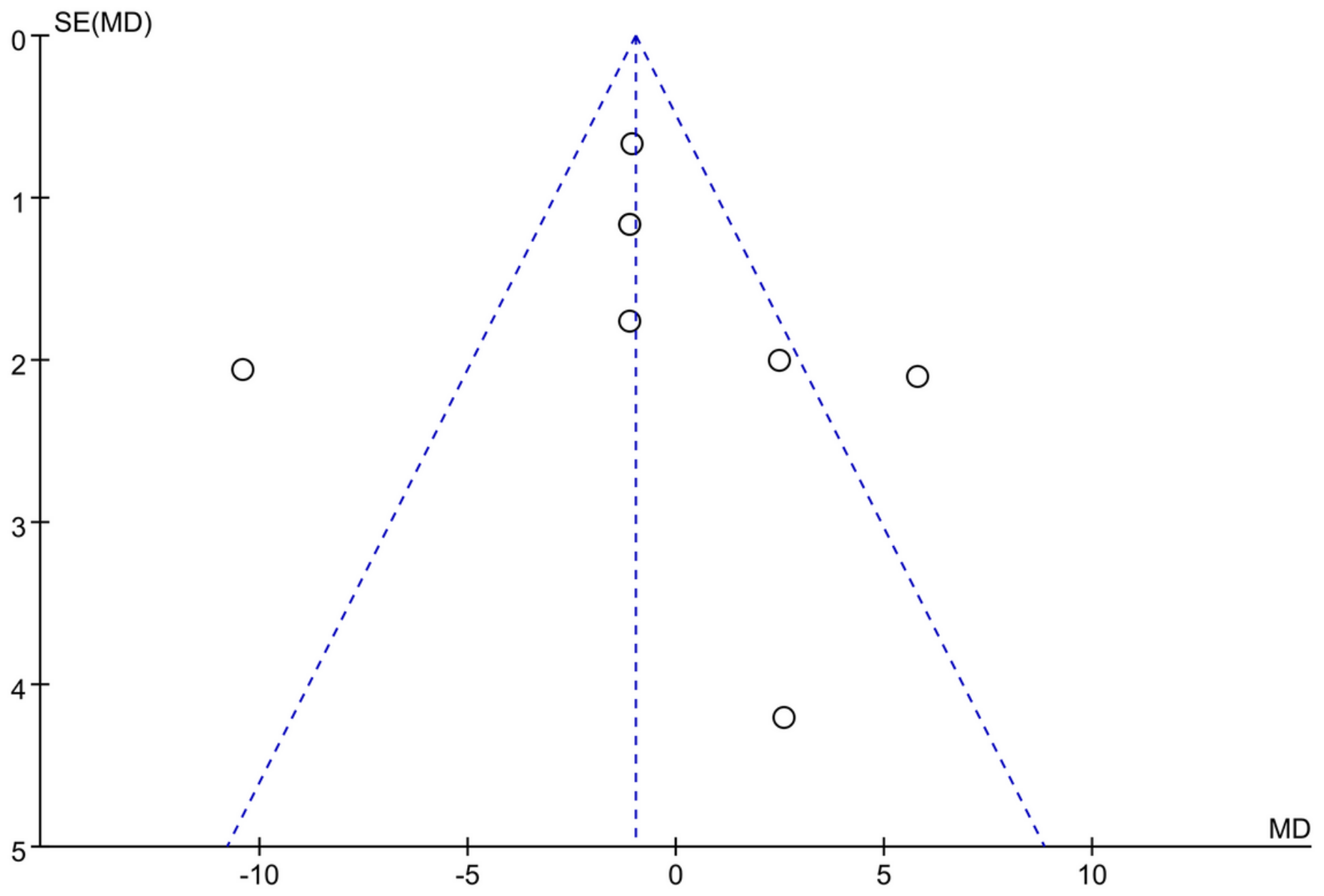

Figure 11

Funnel plot

\section{Supplementary Files}

This is a list of supplementary files associated with this preprint. Click to download.

- Table1.pdf 